Military Technical College Kobry El-Kobba

Cairo, Egypt

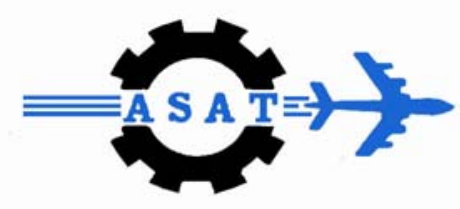

12-th International Conference on

Aerospace Sciences \& Aviation Technology

\title{
A STUDY OF ONE-DIMENSIONAL POWER SYSTEM PARAMETERS EFFECTS ON THE ELECTROMECHANICAL WAVE PROPAGATION
}

\author{
W. Sabry *
}

\begin{abstract}
This paper presents a new idea for studying and analyzing of large electric power systems. The paper supposes that the model is a spatial type, which gives a new approach to model the power system as a continuum model in time and position. Hence, the phenomenon of electromechanical wave propagation and faults spreading throughout the system is appeared. A MATLAB simulation is carried out and the results show the effect of three different power system parameters on the wave propagation: number of generators in the system, internal impedances of these generators and load power factor. These effects were studied and the relation between these parameters and the wave propagation was demonstrated.
\end{abstract}

\section{KEY WORDS}

One-dimensional power system, power system parameters, and electromechanical wave propagation

\footnotetext{
* Egyptian Armed Forces
} 


\section{INTRODUCTION}

From the early years, electric power systems were designed according to the requirements of successful operation. Successful operation of a power system; depends largely on the engineer's ability to provide reliable service to the loads. Ideally, the loads must be fed from constant voltage and frequency sources at all times. In practice this means that both voltage and frequency must be held within tolerances so that the loads may operate satisfactorily [1].

Random faults set the rotors of synchronous machines (which working as large electric generators) in motion with respect to their synchronous frame of reference. The study of this phenomenon is of great interest in electric power engineering, as instability of this motion frequently imposes operational limits on the power system. The normal technique for studying electromechanical dynamic behavior in power systems is to set up a large detailed model of the entire system comprising of nonlinear differential and algebraic equations and then integrate the resulting nonlinear ordinary differential equations numerically [2].

For regular sustained and stable operation, the physical size of the system to be operated will be greatly increased. Systems will become more spatially distributed and the number of generators and buses will increase. Logically, that there are effects in such large systems that are not easily understood using existing models [3].

In large power systems, the transmission lines, generators and loads are considered to be continuous functions of position and time (space). The application of the limit of zero generator internal impedance, with finite rotor inertia and zero transmission line impedance per unit length yields a nonlinear partial differential equation in time and two spatial dimensions for the rotor power angle. This model is called continuum model [2, 4-5].

The equation is a nonlinear version of the standard second-order wave equation, which exhibits expression for the finite wave phase velocity. The continuum technique provides a macro-scale analytical tool to gain an insight into the mechanisms by which the disturbances initiated by faults and other random events propagate in the continuum power system. It is found that the equations of motion can be formulated as a nonlinear partial differential equation on a two-dimensional surface.

This equation is in the form of a wave equation that has a finite propagation speed and exhibits several interesting phenomena related to a continuum power system. Power system engineers have long recognized that disturbances propagate over the power network with finite speed. It is only in recent years that simultaneous measurement of rotor angles, through synchronized phasor measurement units (PMUs), has been made possible with the help of the global positioning system (GPS). Several preliminary observations over long distances have confirmed the existence of a propagation speed well less than that of light [5].

In this paper, the electromechanical wave propagation point of view afforded by the continuum power system and discrete model to explore the effects of some parameters of the system to eliminate or at least, mitigate wave propagation and growing in the 
entire network. These parameters are: number of generators in the power system, internal impedances of these generators and load power factor.

\section{POWER SYSTEM MODEL}

To simulate a power system under the influence of the phenomenon of electromechanical wave propagation, transmission system, generators and loads are modeled with a combination of simultaneous algebraic equations set and other ordinary differential equations set. The continuum principle, which considered for the power system leads to a set of nonlinear partial differential equations. So, the discretization concept was applied in this paper. For the purpose of simulation, the continuum system is discretized in finite number of nodes. The continuum system solution and the descritized continuum solution were consistent with each other [2].

In this paper, one-dimensional discrete ring system with uniform and nonuniform parameters is represented to illustrate electromechanical wave propagation phenomenon. The parameters used in this paper to explain the electromechanical wave propagation characteristics include the number of generators in the system, internal generator transient reactance and loads power factor.

Figure (1) shows the one-dimensional ring system. The system consists of a ring of transmission line sections; each section has certain admittance. The ring consists of a number of nodes (n). At each node, a generator with zero per unit internal impedance and one per unit internal voltage is connected to the node. Also, a shunt load is connected to this node. Figure (2) shows the details of two consecutive nodes.

For the system shown in figure (1) and for each generator, the input mechanical power $\left(P_{m}\right)$, the output electrical power $\left(P_{e}\right)$, the accelerating power $\left(P_{a}\right)$ is the difference between $P_{m}$ and $P_{e}$, the damping constant $\left(D^{\prime}\right)$ and the rotor angle is $(\delta)$. All of these parameters are governed with the normalized swing equation:

$\ddot{\delta}+D^{\prime} \dot{\delta}=P_{a k}$

The symbol $(k)$ means the swing equation of the $k^{\text {th }}$ generator.

\section{SIMULATION RESULTS}

To simulate the proposed system, a 44-generator system simulation was constructed using the MATLAB package. All generators are considered with zero internal impedance and one per unit internal voltage. The normalized damping constant is 0.01 [4]. Also, each shunt load has a magnitude of one per unit and with a 0.8 lagging power factor [6]. The transmission line is supposed to be a pure inductance with an initial value of 0.1 per unit. According to figure (2), $E_{g i}$ represents the internal voltage of generator number (i), $Z_{t l}$ represents the reactance of transmission line section, the term $Z_{g}$ represents the generator internal reactance, and the term $Z_{1}$ represent load at each node. In this simulation, the values taken are: $Z_{\mathrm{tl}}=j 0.1 \mathrm{pu}, Z_{\mathrm{g}}=\mathrm{j} 0.0001 \mathrm{pu}$ and $Z_{\mathrm{I}}=0.8$ + j0.6 pu. 


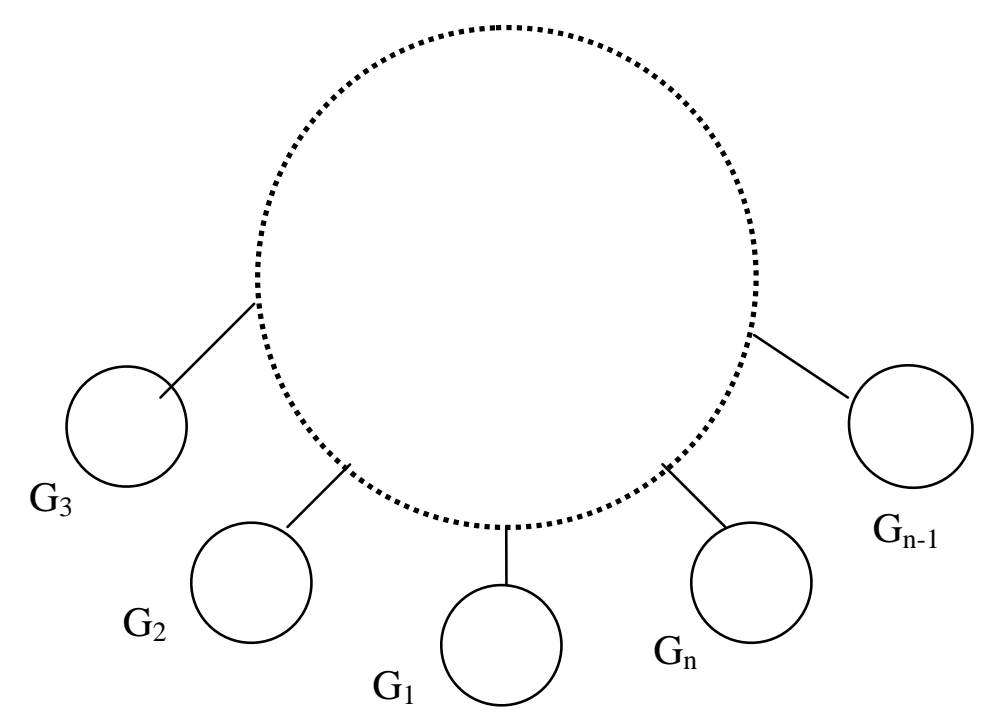

Fig. 1. One-dimensional ring system

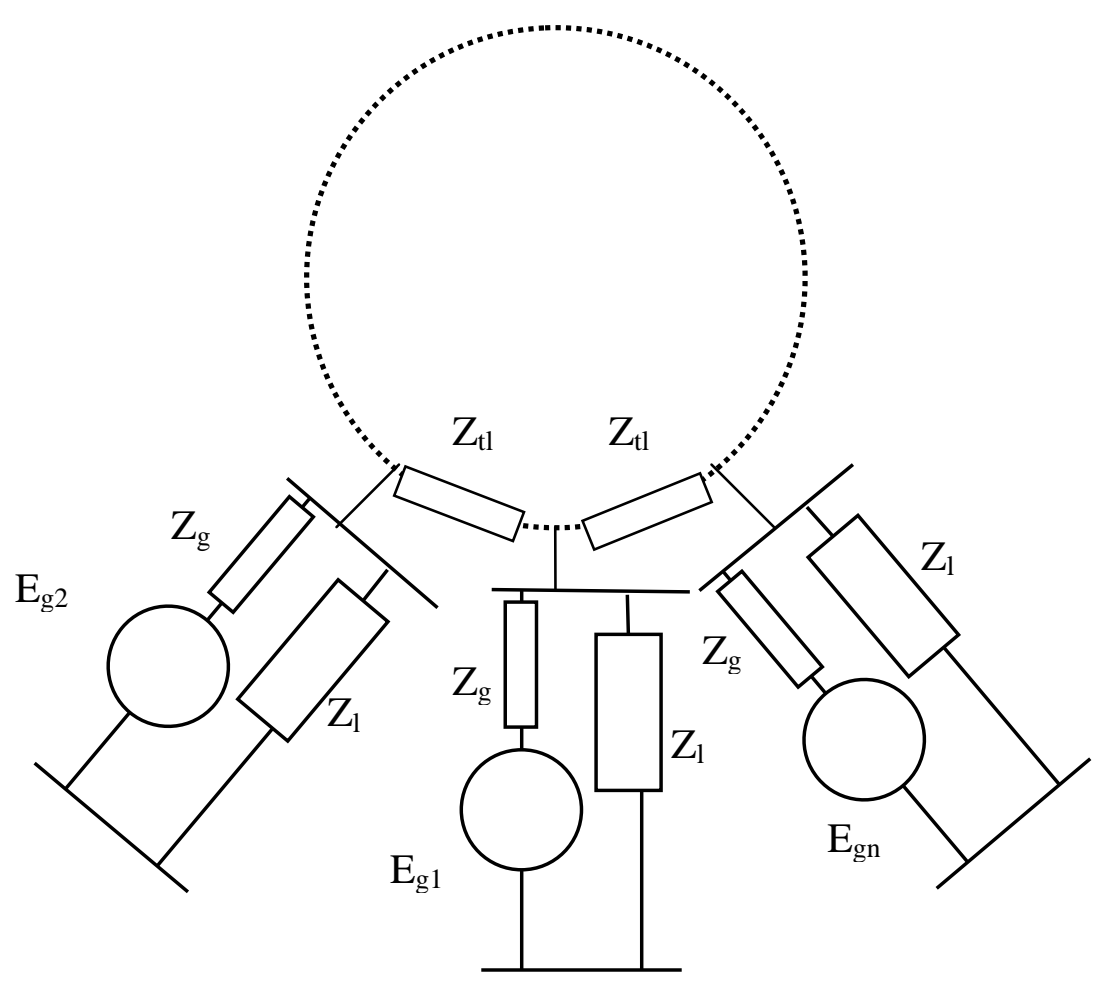


Fig. 2. Two-consecutive nodes

The initial value of each rotor angle is $\delta_{k}=2 \pi \mathrm{k} / 44$, where, $k=1,2 \ldots 44$. The initial perturbation is proposed at the $22^{\text {nd }}$ node (generator terminals) where the disturbance is a smooth function (for example, a one per unit peak value Gaussian distribution of rotor angle displacements in a finite neighborhood of the $22^{\text {nd }}$ machine $\delta_{d}=(1.0) \mathrm{e}^{-0.1(\mathrm{~S}-22)^{2}}$ as shown in figure (3).

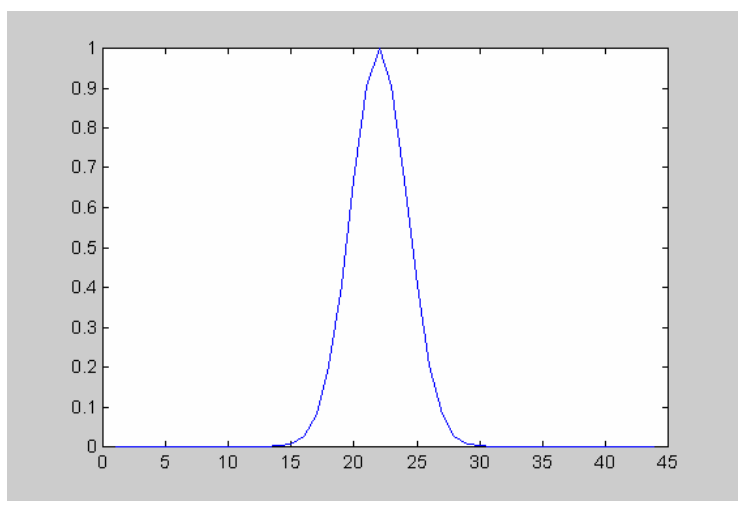

Fig. 3. The initial Gaussian disturbance

Figure (4) illustrates the electromechanical wave propagation of rotor angles with initial Gaussian disturbance centered at the $22^{\text {nd }}$ generator. The wave propagates in both directions. The waves in the direction of the increasing angle grow, while the waves in the reverse direction are attenuated. Figure (5) shows also the behavior of bus voltages with the same initial Gaussian disturbance. It is clear that the voltage is oscillated also, but not disturbed as rotor power angle. In this paper, the voltage behavior is not taken into consideration; all the study is directed towards the behavior and control of rotor power angle.

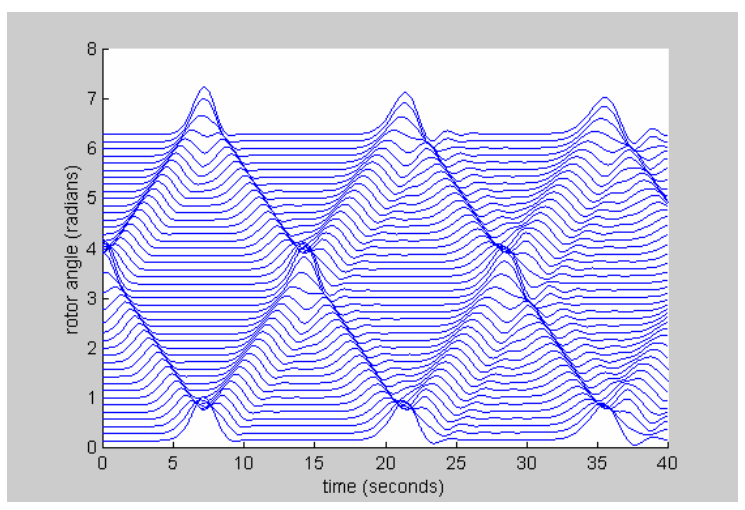

Fig. 4. Rotor angle wave-propagation in the 44-generator system 


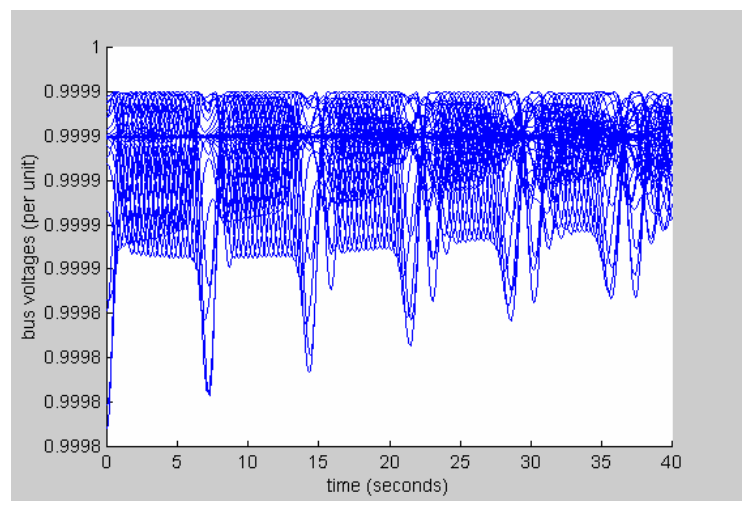

Fig. 5. Bus voltages behavior of the ring system with initial Gaussian disturbance in the 44-generator system

From figure (4), it is noticed clearly the propagation of the electromechanical wave through the entire network. To find the speed of the wave, three selected generators are taken; generator number (1), (22) and (43) as shown in figure (6). From generators (1), (22) and (43), the peak of the $1^{\text {st }}$ generators occurs at about 7.12 seconds, the peak of the $22^{\text {nd }}$ generators occurs at about 14.295 seconds and peak of the $43^{\text {rd }}$ generators are about 21.36 seconds.

This gives us information about the wave speed between the $1^{\text {st }}$ and $22^{\text {nd }}$ generators, which are calculated from the simple formula of the distance by the time. The distance here is the distance between the $1^{\text {st }}$ and $22^{\text {nd }}$ which is 21 per unit length taken between two adjacent generators, and the time is the difference between the time of occurrence of the peaks of the $1^{\text {st }}$ and $22^{\text {nd }}$ generators which is ( $14.295-7.12=7.175$ seconds), so the wave speed is $21 / 7.175$ which equals 2.9268 per unit length taken between two adjacent generators per seconds. The same is applied between the $22^{\text {nd }}$ and $43^{\text {rd }}$ generators, which give wave speed of 2.9723 per unit length taken between two adjacent generators per seconds. So the average of the two speeds can be taken to say that the electromechanical wave speed of the described case is 2.9495 per unit length taken between two adjacent generators per seconds.

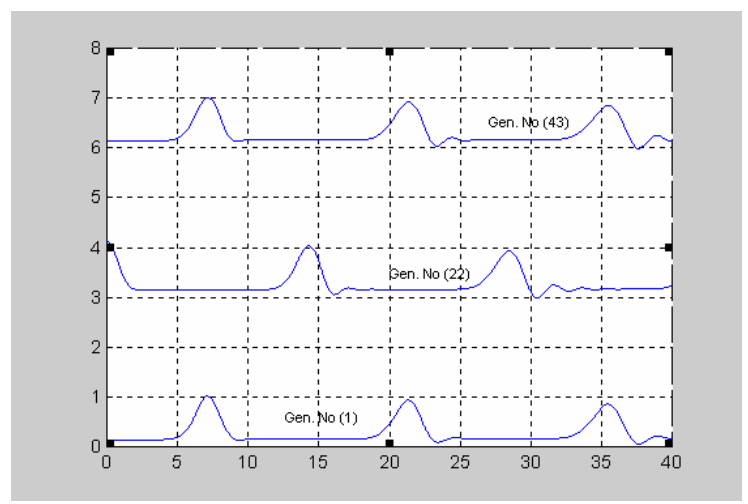

Fig. 6. Rotor angle wave-propagation for a selected three generators in the 44generator system 
There is another way to explain the propagation speed, that is the distance between the $22^{\text {nd }}$ and the $43^{\text {rd }}$ generator is 21 per unit length taken between two adjacent generators and the peak of the wave of the $22^{\text {nd }}$ generator occurs at time zero and the first peak of the wave at the $43^{\text {rd }}$ generator occurs at 7.12 seconds, so the speed will be $21 / 7.12=$ 2.9494 per unit length taken between two adjacent generators per seconds.

Another characteristic of the electromechanical wave is the frequency of the wave. This can be presented from figure (6) for any of the three selected generators by calculating the difference of time of peak occurrence for the specified generator, this give us the period of the wave, from which the frequency of the wave is calculated. Let's take the $1^{\text {st }}$ generator, first peak occurs at 7.12 seconds and the successive peak occurs at 21.33 seconds, so the period of the wave is 14.21 seconds and the frequency of the electromechanical wave is 0.07 cycle per second. So, the above characteristics of the electromechanical wave are calculated for the specified data given, and if the data is changed these characteristics will certainly be changed.

\subsection{Effect of number of generators on wave propagation}

The case of 44-generators as mentioned above, the speed of electromechanical wave propagation was 2.9495 per unit length taken between two adjacent generators per seconds. If the ring system has 64-generators and the disturbance occurrence at the middle of the generators (i.e. at the $32^{\text {nd }}$ generator) and all the other data maintained constant.

The wave propagation is shown in figure (7), and the wave speed and frequency is calculated from figure (8). The peak of the $1^{\text {st }}$ generator occurs at about 10.34 seconds, the peak of the $32^{\text {nd }}$ generators occurs at about 20.67 seconds and peak of the $63^{\text {rd }}$ generators are about 30.92 seconds. The wave speed as calculated before is 3.024 per unit length taken between two adjacent generators per seconds. This result gives us indication that the wave propagation speed increase with dense power systems.

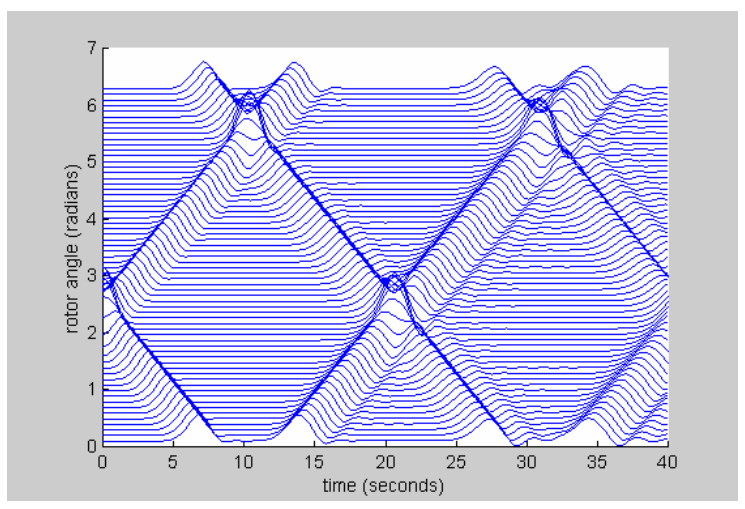

Fig. 7. Rotor angle wave-propagation in the 64-generator system with Gaussian disturbance at $32^{\text {nd }}$ generator 


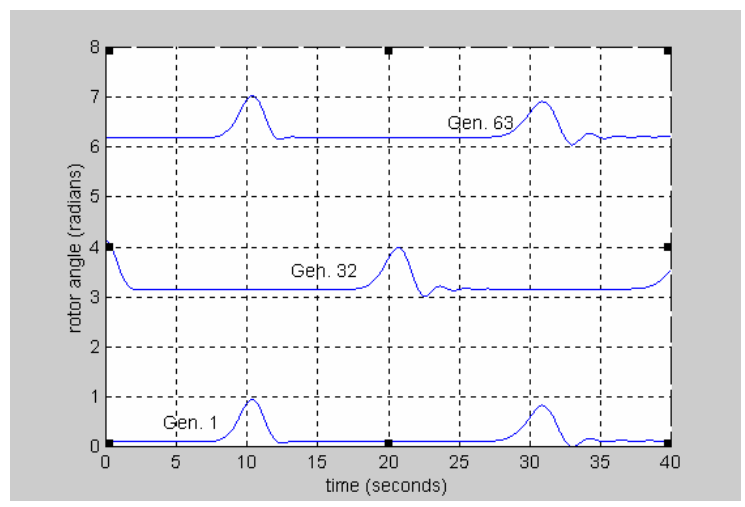

Fig. 8. Rotor angle wave-propagation for a selected three generators in the 64generator system

\subsection{Effect of generator internal impedance on wave propagation}

The generators internal impedance has a great effect on the power network stability and on the propagation of the electromechanical wave. This effect can be explained by changing only the value of generators internal impedance and see the behavior of the electromechanical wave and hence the power network stability.

For Case (1), generator internal impedance equal to $0.001 \mathrm{pu}$, this is shown in figures (9) and (10) which show the wave propagation for 44-generator of ring system and a selected three generators to measure the wave speed and frequency. From figure (10) the $1^{\text {st }}$ peak of the wave of $43^{\text {rd }}$ generator occurs at 7.18 seconds, so the speed of propagation is 2.9248 per unit length taken between two adjacent generators per seconds. The frequency can be calculated from the $2^{\text {nd }}$ peak occurrence of the $22^{\text {nd }}$ generator, which occurs at 14.33 seconds, so the frequency of the electromechanical wave for 0.001 pu generator internal impedance is 0.0698 cycle per second.

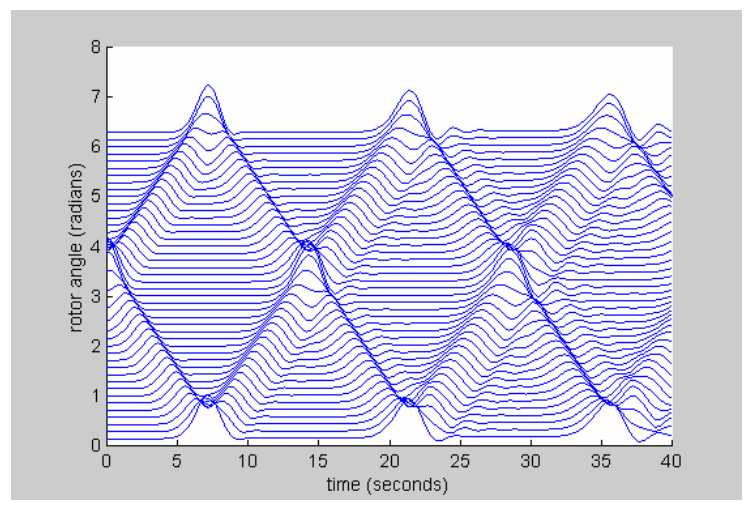

Fig. 9. Rotor angle wave-propagation in the 44-generator system with generator internal impedance equal $0.001 \mathrm{pu}$ 


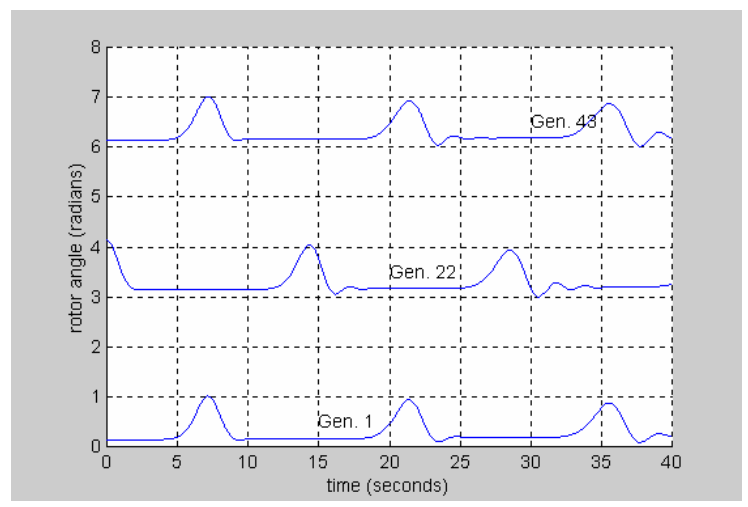

Fig. 10. Rotor angle wave-propagation for a selected three generators in the 44generator system with generator internal impedance equal $0.001 \mathrm{pu}$

For the case (2), the generator internal impedance equal $0.01 \mathrm{pu}$, the wave propagation is shown in figures (11) and (12). From figure (12) the $1^{\text {st }}$ peak of the wave of $43^{\text {rd }}$ occurs at 7.4 seconds, so the speed of propagation is 2.838 per unit length taken between two adjacent generators per seconds. The frequency also can be calculated from the $2^{\text {nd }}$ peak occurrence of the $22^{\text {nd }}$ generator, which occurs at 14.68 seconds, so the frequency of the electromechanical wave for 0.01 pu generator internal impedance is 0.0681 cycle per second. In case (3), the generator internal impedance is increased to $0.05 \mathrm{pu}$, the wave propagation is shown in figures (13) and (14). This will not only affect the speed and frequency of the wave but also affect the stability of the system. It is shown from figures (13), (14) that waves of rotor angle increase indefinitely and this means that the rotor angle does not return to equilibrium value after disturbance, so the system will be unstable for this case with these parameters.

From the above three cases: it is noticed that the increase of the generator internal impedance decreases the speed and frequency of the rotor angle electromechanical wave, but if the generator internal impedance increases above certain value the power system will become unstable. This value of generator internal impedance is determined depending on the power network parameters.

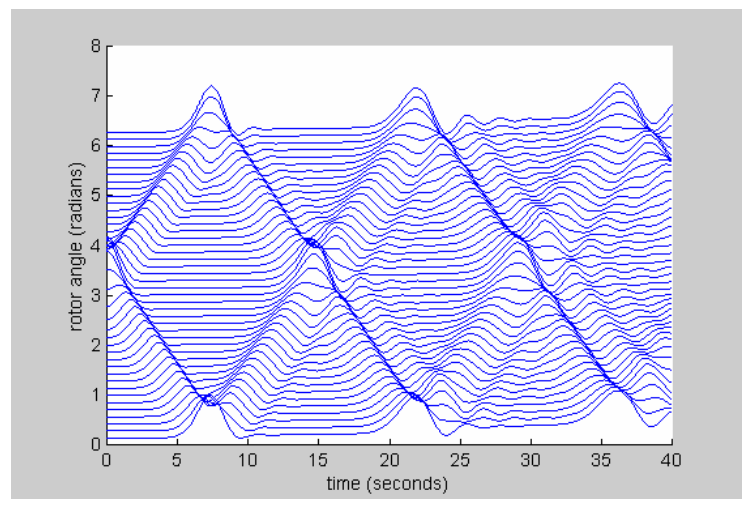

Fig. 11. Rotor angle wave-propagation in the 44-generator system with generator internal impedance equal $0.01 \mathrm{pu}$ 


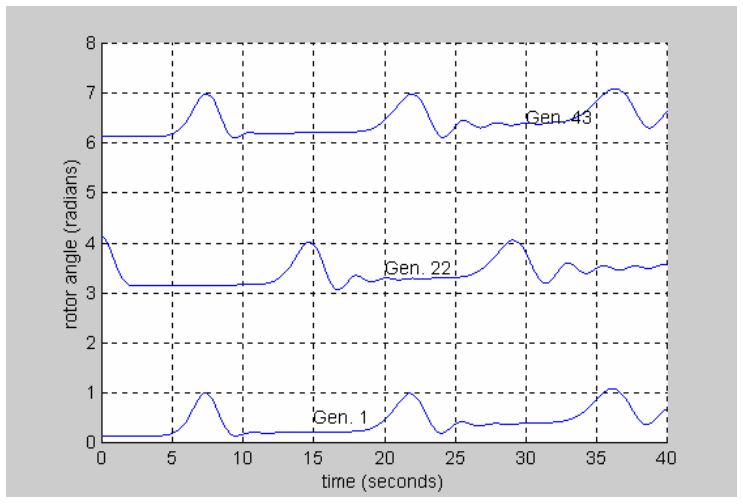

Fig. 12. Rotor angle wave-propagation for a selected three generators in the 44generator system with generator internal impedance equal $0.01 \mathrm{pu}$

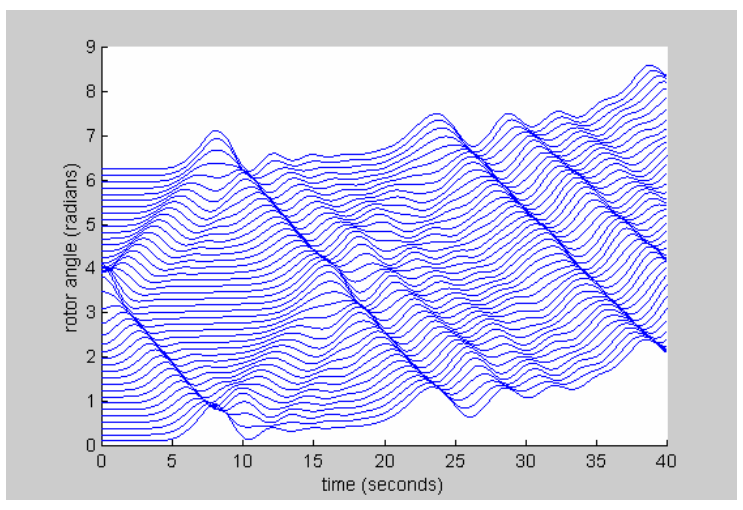

Fig. 13. Rotor angle wave-propagation in the 44-generator system with generator internal impedance equal $0.05 \mathrm{pu}$

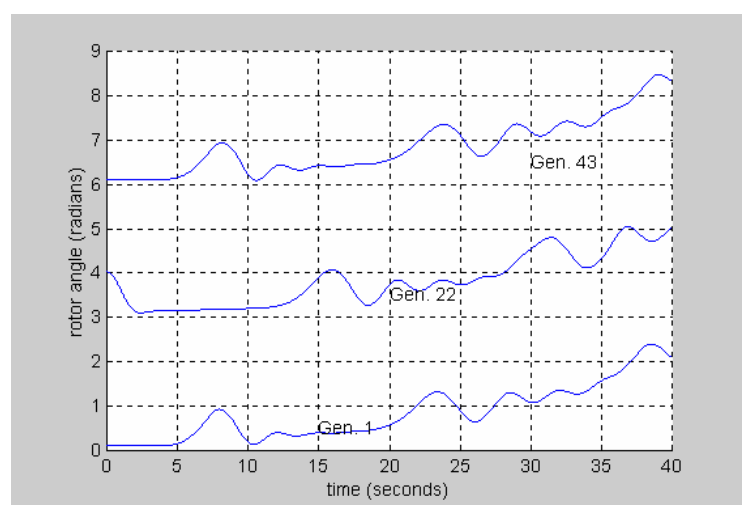

Fig. 14. Rotor angle wave-propagation for a selected three generators in the 44generator system with generator internal impedance equal $0.05 \mathrm{pu}$ 


\subsection{Effect of load power factor on wave propagation}

The power factor of the load is studied to show its effect on the propagation of the electromechanical wave. The magnitude of the load impedance is taken to be one per unit in all cases of study. The studying is pointed at the nature of the load, i.e., pure resistance, pure inductance, pure capacitance, lead and lag power factor.

To examine the effect of power factor we present the different cases as follows:

The first case when the load has lag power factor of 0.8 , which is shown in figure (15). In figure (16) the power factor of the load is 0.8 lead and the loads of pure inductance, pure capacitance, and pure resistance are shown in figures (17), (18) and (19) respectively

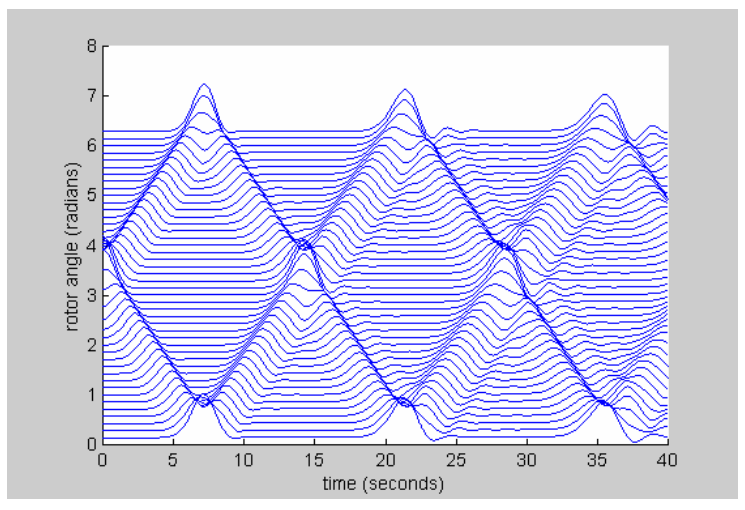

Fig. 15. Rotor angle wave-propagation in the 44-generator system with the load has lag power factor of 0.8

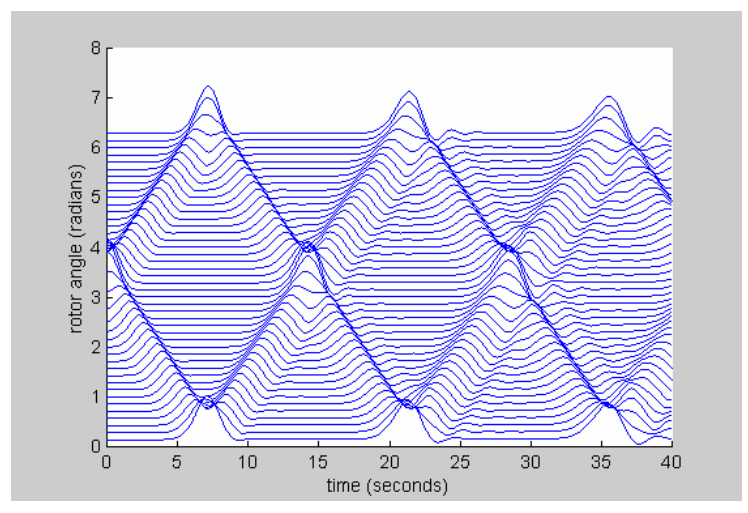

Fig. 16. Rotor angle wave-propagation in the 44-generator system with the load has lead power factor of 0.8 


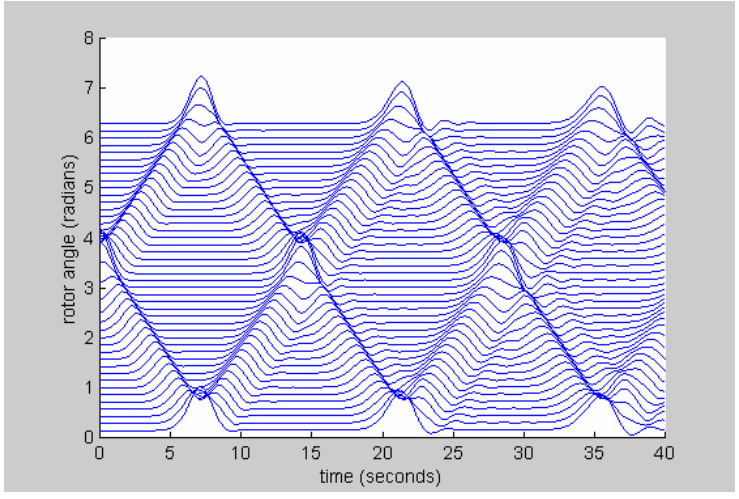

Fig. 17. Rotor angle wave-propagation in the 44-generator system with the load of pure inductance

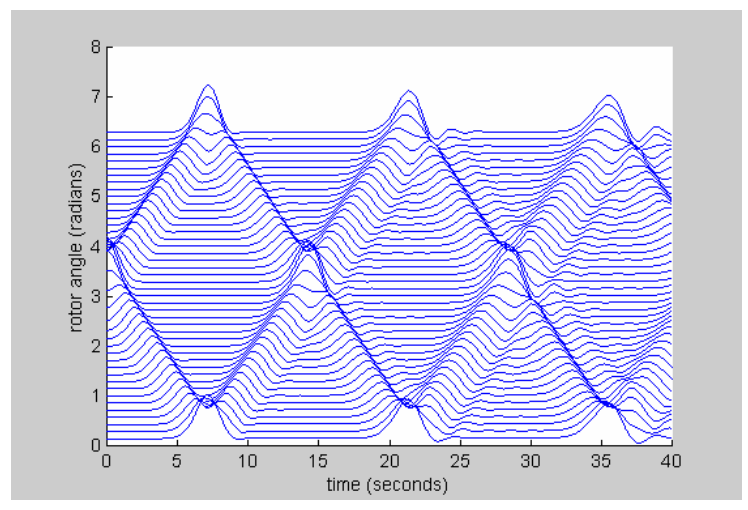

Fig. 18. Rotor angle wave-propagation in the 44-generator system with the load of pure capacitance

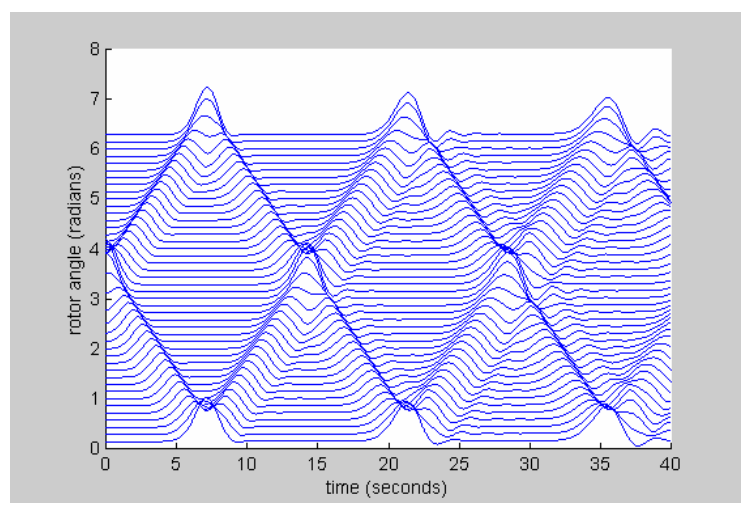

Fig. 19. Rotor angle wave-propagation in the 44-generator system with the load of pure resistance 
From the above figures, it is shown for a large extent that the power factor change doesn't affect the electromechanical wave propagation. This can be interpreted that as the magnitude of load impedance is still constant the magnitude of its current is also still constant (where the internal voltage is held constant at 1 pu., and the generator internal impedance is zero). As the magnitude of the load current doesn't change with varying the load power factor the magnitude of the generator output current doesn't change also. The magnitude of the generator output current has the mainly effect on the output electrical power, which affects the behavior of the electromechanical wave propagation.

\section{CONCLUSION}

This paper presents a new idea for studying and analyzing of large electric power systems. The study gives a new approach to model the power system as a continuum model. Hence, the phenomenon of electromechanical wave propagation and faults spreading is discussed. The MATLAB package is used to simulate power system in case of ring one-dimensional system. The simulation results show the consequences of electromechanical wave propagation on power system. Also, the effect of three different parameters on the wave propagation (number of generators in the system, internal impedances of these generators and load power factor) was studied and the correlation between these parameters and the propagation was demonstrated.

From the discussion of previous sections and previous work [7-8], the electromechanical wave propagation has harmful effects on power systems; of these effects is the out of synchronization of some system machines, or the overall system going in black out. In some cases, the electromechanical wave propagation is selfdamped in sustain with the existing system parameters. In other cases, the system is accelerated into the instability situation. In both cases, the damping of wave propagation is intended and controllers must be taken into consideration.

\section{REFERENCES}

[1] Anderson, P. M. and Fouad, A. A., "Power System Control and Stability", Galgotia publisher, India, (1984).

[2] Thorp, J. S., Seyler, C. E. and Phadke, A. G., "Electromechanical Wave Propagation in large Electric Power Systems", IEEE Transactions on Circuits and Systems I: Fundamental Theory and Applications, Vol. 45, No. 6, p 614-622, June (1998).

[3] Kundur, P., "Power System Stability and Control", EPRI Power System Engineering Series, McGraw-Hill, (1994).

[4] Thorp, J. S., Seyler, C. E., Parashar, M. and Phadke, A. G. "The Large Scale Electric Power System as a Distributed Continuum", IEEE Power Engineering Review, p 49-50, January (1998).

[5] Parashar, M., Thorp, J. S. and Seyler, C. E. "Continuum Modeling of Electromechanical Dynamic in Large-Scale Power Systems", IEEE Transactions on Circuits and Systems I: Regular Papers, Vol. 51, No. 9, p 1848-1858, September (2004). 
[6] Liling Huang, Manu Parashar, Phadke, A. G. and James S. Thorp "Effects of Electromechanical Wave Propagation on Protection Systems", International Conference on Power Systems and Communication Systems Infrastructures for the Future, Section II, No. 3, Beijing, Sep. (2002).

[7] Sabry, W., Mansour, W. M., Salama, M. M., Saied, E. M. and Abuserie, M. E., "Effect of Transmission Line Impedance on One-Dimensional Ring Power System Electromechanical Wave Propagation", Al-Azhar University Journal (AUEJ), Vol. 9, No. 2, p 396-403, Apr. (2006).

[8] Sabry, W., "Effect of Damping Constant and Rotor Inertia Constant On OneDimensional Ring Power System Electromechanical Wave Propagation", 5th International Conference on Electrical Engineering ICEENG 2006, p EP2/1EP2/11, 16-18 May (2006). 